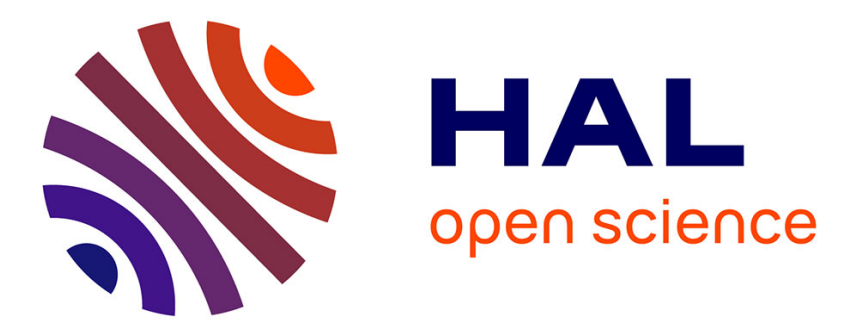

\title{
Flat lens for pulse focusing of elastic waves in thin plates
}

\author{
Marc Dubois, M. Farhat, E. Bossy, S. Enoch, S. Guenneau, P. Sebbah
}

\section{To cite this version:}

Marc Dubois, M. Farhat, E. Bossy, S. Enoch, S. Guenneau, et al.. Flat lens for pulse focusing of elastic waves in thin plates. Applied Physics Letters, 2013, 103 (7), pp.071915. 10.1063/1.4818716. hal-01680699

\section{HAL Id: hal-01680699 https://hal.univ-antilles.fr/hal-01680699}

Submitted on 6 Jun 2019

HAL is a multi-disciplinary open access archive for the deposit and dissemination of scientific research documents, whether they are published or not. The documents may come from teaching and research institutions in France or abroad, or from public or private research centers.
L'archive ouverte pluridisciplinaire HAL, est destinée au dépôt et à la diffusion de documents scientifiques de niveau recherche, publiés ou non, émanant des établissements d'enseignement et de recherche français ou étrangers, des laboratoires publics ou privés. 


\title{
Flat lens for pulse focusing of elastic waves in thin plates
}

\author{
M. Dubois ${ }^{1}$, M. Farhat ${ }^{2,3}$, E. Bossy ${ }^{1}$, S. Enoch ${ }^{2}$, S. Guenneau², P. Sebbah ${ }^{1}$ \\ ${ }^{1}$ Institut Langevin, ESPCI ParisTech, CNRS 7587, 1 Rue Jussieu, 75238 Paris Cedex 05, France. \\ ${ }^{2}$ Aix-Marseille Université, CNRS, Ecole Centrale Marseille, Institut Fresnel, 13013 Marseille, France \\ ${ }^{3}$ Institute of Condensed Matter Theory and Solid State Optics, Abbe Center of Photonics, Friedrich-Schiller- \\ Universität Jena, D-07743 Jena, Germany
}

The ability of left-handed materials to overcome the diffraction limit was first considered as one of the most exciting and challenging outcome of the negative refraction concept. Flat lens focusing of elastic waves is however a challenge. We demonstrate broadband focusing of elastic waves at $10 \mathrm{kHz}$ carrier frequency, below the first stop band, in a $45^{\circ}$-tilted square array of circular air holes perforated in a Duraluminium thin plate. By adjusting the relative thickness of the outer plate we achieve large-angle negative refraction with diffraction-limited lateral resolution. We find good agreement with a simple beam-lattice model and finite-difference time-domain simulations. 


\section{INTRODUCTION}

Veselago's 1968 discovery of a convergent flat lens via negative refraction ${ }^{1}$ and Pendry's 2000 bold claim that this lens has a resolution only limited by its material constituents ${ }^{2}$ have paved the way towards an enhanced control of light trajectories in materials structured on a sub-wavelength scale. The subsequent experimental demonstration of negative refraction of microwaves in a metamaterial consisting of a periodic arrangement of thin straight wires and thin loops ${ }^{3}$ fueled the interest in the physics of negative refraction ${ }^{4}$.

These discoveries prompted physicists to also explore focusing via negative refraction in the context of resonant discrete spring-mass mechanical systems for other types of waves, such as acoustic or elastodynamic waves, where the effective dispersive parameters could be Young's modulus, density, bulk modulus and so forth ${ }^{5-9}$.

In the tracks of photonics, phononic crystals ${ }^{10}$ have also been proposed as an alternative to resonant acoustic metamaterials. Dispersion bands can be tailored to force group and phase velocities to point in opposite directions. Experiments in linear surface water waves ${ }^{11}$ and sonic wave $\mathrm{e}^{12-16}$ transmission through water-immersed pillars, or layers of perforated plates ${ }^{17}$, illustrate the high potential of acoustic metamaterials.

However, when one moves into the area of elastodynamic waves, governing equations become much less tractable as compressional and shear waves are inherently coupled in structured solids. Another difficulty intrinsic to the tensor nature of the Navier's equations is that they do not behave nicely upon a change of coordinates ${ }^{18}$. In contrast to Maxwell's equations, their form is not preserved under geometric transforms such as space folding, whereby the image plane of a flat lens with a negative refractive index is expected to map onto its source plane ${ }^{19}$. 
There is however a limiting geometry where flat lens focusing for elastodynamics waves has been recently predicted in a time-harmonic setting ${ }^{20}$ : a vibrating thin plate. Here, compressional and shear components of the wave are linked by stress-free boundary conditions on the limiting surfaces to produce dispersive Lamb modes, leading to simplification of the tensor equations into a fourth order differential operator such as those of Timoshenko or Graff ${ }^{21,22}$. Beyond the interest in diffractive tomography ${ }^{23}$ and non-destructive testing ${ }^{24}$ of large plate-like structures, control of flexural waves ${ }^{25}$ in structured plates has become an increasingly fashionable topic in the past few months with the first experimental validation ${ }^{26}$ of the theoretical proposal for cloaking in thin plates $^{27,28}$. Recently, negative refraction in elastic plates was reported at an interface via mode conversion ${ }^{29}$ or across a prism-shaped phononic crystal ${ }^{30}$. Advantage was taken from band folding in the second band where the directions of the wave vector and group velocity are anti-parallel, the drawback being to work at high frequencies, where several acoustic modes coexist and no manageable asymptotic theory seems to be available. However, large-angle negative refraction (LANR) and flat lens focusing for bending waves have never been reported to date.

Here, we show that LANR and focusing of elastic waves with resolution down to the diffraction limit are within a relatively easy reach in structured thin plates, in the first band (acoustic band) where homogenization theory is valid and analytical form of the band diagram is derived. Inspired by the earlier work by Joannopoulos's group for electromagnetic waves ${ }^{31}$, we consider a flat lens formed by a square array of circular air holes tilted through an angle of $45^{\circ}$ perforated in a Duraluminium thin plate. By matching free-space and platonic crystal dispersion curves, broadband LANR is achieved. Pulse measurements allow to discriminate between direct focusing and spurious reflections from the edges of the plate. Three-dimensional finite-difference timedomain simulations including open boundary conditions confirm the experimental observation. 


\section{THEORY AND NUMERICS}

We first compute the equifrequency contour and the band structure (Fig. 1) of the elastodynamic equations in a platonic crystal: A transversely infinite thin plate structured with a square lattice of see-through holes. We use Finite Element Method by enforcing Floquet-Bloch conditions on the opposite vertical boundaries of an elementary cell (a thin box) containing a see-through stress-free circular cylindrical hole and stress-free top and bottom boundaries (see right inset in Fig.1b). The effect of the crystal anisotropy and its dependence on geometric parameters (hole radius, $r$, lattice constant, $l$, plate thickness, $h$ ) is well understood by the graphical representation of the isofrequency surfaces (Fig. 1a), which describe the variation of the wave vector with direction and frequency. Isofrequency contours of the lowest dispersion curve (in red in Fig. 1b) display the hallmark of LANR in the $\Gamma$-M direction: isofrequency surfaces become convex everywhere about the M-point in the reciprocal space, and the size of the isofrequency contours shrinks with increasing frequency. The direction of energy transport, determined by the group velocity, $v_{g}$, which is perpendicular to the isofrequencies, points inward around the M-point (Fig. 1d), as opposed to outward around the $\Gamma$-point at lower frequencies ${ }^{32}$.

Remarkably, we find that the acoustic band can be approximated in closed form if one considers that the flexions of a periodically perforated plate can be mimicked by the flexions of a lattice of beams33 (see left inset in Fig.1b). Floquet-Bloch bending waves propagating in such a lattice are described by the dispersion relation ${ }^{20,33}$ :

$\sin (\beta l)\left[\cos \left(k_{1} l\right)+\cos \left(k_{2} l\right)-2 \cos (\beta l)\right]\left[\cos \left(k_{1} l\right)+\cos \left(k_{2} l\right)-2 \cosh (\beta l)\right]=0$

with $2 l$ the pitch of the array and $k_{1}$ and $k_{2}$ the components of the Bloch vector $\boldsymbol{k}$. We define the

reduced frequency $\beta$ with $\beta^{2}=p \sqrt{\rho h / D}$ with $p$ the pulsation, $D=E h^{3} / 12\left(1-v^{2}\right)$, the flexural rigidity, $v$ the Poisson's ratio, $E$ the Young's modulus, $h$ the plate thickness and $\rho$ the plate density. 
The second factor in this equation provides us with the dashed curves in Fig. 1b. The match between asymptotics and numerics computed by finite elements method (solid curves in Fig. 1b) is sharp in the acoustic band. Beyond the acoustic band, the asymptotic model breaks down when the flexural wave starts sensing the specific shape of air holes, i.e. when we move away from the homogenization regime.

To demonstrate LANR and flat lens focusing, we now consider a rectangular lens formed by a $45^{\circ}$ tilted ( $\Gamma-\mathrm{M}$ direction) finite-sized section of the platonic crystal perforated in a larger thin plate. The lens interface, which is optimally oriented with respect to the $\Gamma$-M direction, introduces a mismatch between the isofrequency contours outside and within the platonic crystal (blue and red contours in Fig. 1a) at the working frequency where negative refraction is expected (above the Xpoint). Nevertheless, better adjustment of isofrequency contours is still possible by taking advantage of an extra degree of freedom offered by the plate geometry: The "free-space" bendingwave dispersion relation ${ }^{34}$ is independently adjustable by varying the thickness $h$ of the plate (with $h<<\lambda$ ). The effect of increasing the plate thickness outside the platonic crystal is seen in Fig. 1b where the point at crossing of the two dispersion curves is brought closer to the working frequency. Consequently, negative refraction operates on a larger angle ${ }^{32}$.

\section{EXPERIMENTS}

A $2 \mathrm{~mm}$-thick Duraluminium rectangular plate ${ }^{35}(500 \mathrm{~mm}$ x $300 \mathrm{~mm})$ was first thinned down to $1 \mathrm{~mm}$ in its central region $(98 \mathrm{~mm}$ x $300 \mathrm{~mm})$. A regular square lattice of 104 circular holes with radius $6 \mathrm{~mm}$ was then computer-aided perforated in this region. The structured plate is shown in the inset of Fig. 3. The platonic crystal has a lattice constant $l=15 \mathrm{~mm}$, resulting in a surface filling-fraction of $50 \%$ and rectangular dimensions $L=98 \mathrm{~mm} \times H=234 \mathrm{~mm}$. The square lattice 
orientation is $45^{\circ}$ with the edges of the plate ( $\Gamma-\mathrm{M}$ direction). The edges of the plate are covered by a $2 \mathrm{~mm}$-thick layer of blue-tack on both sides over $2 \mathrm{~cm}$, which turns out to be a good absorber in the $\mathrm{kHz}$ range. $\mathrm{A} 3 \mathrm{~dB}$ attenuation of the reflected wave at the edges is thus achieved, which is enough to observe focusing of a short pulse before edge reflections become significant.

Phenyl salicylate (Salol-melting point of $43^{\circ} \mathrm{C}$ ) is used to bond and couple a $1 \mathrm{~cm}$-diameter piezoelectric ceramic pastille (PKS1-4A1 MuRata Shock Sensor) on the plate along its main median at a distance $L / 5=1.8 \mathrm{~cm}$ from the lattice. A digitized acoustic Gaussian pulse centered at $f_{0}$ with bandwidth $\sigma$ is digital-to-analog converted and amplified before being emitted by the pastille. In the $\mathrm{kHz}$ range, only zero-order symmetric, $S_{0}$, and anti-symmetric, $A_{0}$, modes can propagate in the plate ${ }^{22}$.

On the other side of the plate, the acoustic field velocity, $v(\boldsymbol{r}, t)=A(\boldsymbol{r}, t) \cos \left(2 \pi f_{0} t+\varphi(\boldsymbol{r}, t)\right)$, is measured at every point at the surface of the plate by a laser vibrometer (Polytec sensor head OFV505, controller OFV5000). The vibrometer is only sensitive to vertical-displacement velocity and therefore measures preferentially the anti-symmetric mode A0. An X-Y stage allows scanning of the probe across the plate, including the crystal. The spatial resolution of the scan is $2 \mathrm{~mm} \times$ $2 \mathrm{~mm}$. The vertical-displacement velocity, $v(\boldsymbol{r}, t)$, is measured and the slowly varying envelope, $A(\boldsymbol{r}, t)$, and phase, $\varphi(\boldsymbol{r}, t)$ are extracted using the Hilbert transform.

The amplitude-squared, $|A(\boldsymbol{r}, t)|^{2}$ and the phase $\varphi(\boldsymbol{r}, t)$ of the velocity field at $t=833 \mu$ s are shown in Fig. 2 for carrier frequencies $f_{0}=5 \mathrm{kHz}$ and $10 \mathrm{kHz}$. For a pulse with $f_{0}=5 \mathrm{kHz}$ and $\sigma=3.0$ $\mathrm{kHz}$, the divergent wavefront issued from the source is seen to traverse the platonic crystal without much distortion (Fig. 2a,b). This is consistent with the corresponding isofrequency, which surrounds the $\Gamma$-point. Above the X-point, at $f_{0}=10 \mathrm{kHz}$, propagation operates a dramatic change (fig. 2c,d): A convergent wavefront emerges from the platonic crystal and the acoustic pulse 
refocuses at a distance $0.4 L$, where $L$ is the lens thickness, and reaches its peak value at $t=833 \mu$ s, $348 \mu$ s after the initial pulse, which corresponds to the travel time within the periodic lattice. The lensing effect is best illustrated in the movie ${ }^{36}$ where the time evolution of the slowly varying envelope squared $|A(\boldsymbol{r}, t)|^{2}$ of the velocity-field crossing the sample is shown for an acoustic pulse centered at $f_{0}=10 \mathrm{kHz}$ and $\sigma=2.3 \mathrm{kHz}$. The contrast is blatant in the phase representation between the diverging propagating wavefront at $f_{0}=5 \mathrm{kHz}$ (Fig. 2a) and the wavefront emerging from the focal point at $f_{0}=10 \mathrm{kHz}$ (Fig. 2d). We define the lateral resolution as the full width at half maximum (FWHM) of the lateral amplitude-profile of the image spot. A lateral resolution of 0.59 $\lambda$ is achieved (Fig. 3, open circles), where $\lambda=44.7 \mathrm{~mm}$ is the wavelength of Lamb waves in the plate at $10 \mathrm{kHz}$. A comparison of the maximum amplitude at the focal point, $A_{F}$, and at the first side lobes, $A_{S L}$, gives a lateral contrast $\left|A_{F^{-}} A_{S L}\right| /\left|A_{F}+A_{S L}\right|=0.63$. Interestingly enough, significant transmission, $A_{F} / A_{S}=27 \%$, is achieved, where $A_{S}$ is the maximum amplitude at the source position. To assess the effect of the double-step geometry, we reproduce the experiment in a plate with identical platonic structure but uniform thickness of $1 \mathrm{~mm}$. In this case, the resolution at focus is no better than $1.05 \lambda$ (Fig. 3, open diamonds). This confirms that the double-step geometry we use to better match the equifrequency contours indeed significantly improves the LANR. Focusing is also observed with a source displaced laterally from the median (x-axis) by $L / 2$, which rules out any symmetry spurious effect which might result from residual reflections.

The experimental results were systematically compared to three-dimensional Finite Difference Time Domain (3D-FDTD) numerical simulations ${ }^{37,38}$. The effect of air loading was neglected in the simulations. The plate was assumed to stand in vacuum: Stress-free boundary conditions were applied on the upper and lower surfaces of the plate, and vacuum within the simulation domain was simply modeled by a material with vanishing elastic constants. Initially, $3 \mathrm{~dB}$-absorbing 
boundary layers (ABL) $)^{39}$ were used to reproduce the experimental conditions. The numericallycomputed intensity and phase distributions are shown in Fig. 2e,f, and the lateral profile is given in Fig. 3 (dashed line). The similarity with experimental data is striking for the intensity and phase spatial distribution at $t=833 \mu \mathrm{s}$, as well as for the lateral resolution $(0.61 \lambda)$. Numerical simulations were reproduced using highly absorbing boundary layers with $54 \mathrm{~dB}$ attenuation to simulate a transversely unbounded plate and test the dependence of the refocusing resolution on the boundary conditions. We confirm that multiple reflections at the edges of the plate do not artificially contribute to the lateral resolution before the pulse is refocused.

We find that focusing is effective from $8 \mathrm{kHz}$ to $12 \mathrm{kHz}$ leading to broadband lensing effect with $\Delta f / f_{0}=40 \%$. The quality of the focus only degrades at higher frequencies where isofrequency contours shrink around the M-point whereas the free-space isofrequency circle increases: The larger mismatch between the two contours reduces the angular aperture of the refocused beam.

\section{CONCLUSION}

In conclusion, we have theoretically investigated and experimentally demonstrated focusing of elastic waves in a thin plate by a flat lens simply realized with a square array of circular see-through holes. The fact that focusing occurs at a frequency in the homogenization domain (i.e. deep down on the acoustic band) allows for an accurate asymptotic estimate by mimicking the dynamics of the structured plate by that of flexural waves propagating within a lattice of beams. We point out that on average, the shape of holes does not affect the lower part of the acoustic band, which is obviously no longer the case when one moves towards higher frequencies. Working at low frequencies is advantageous since a single Lamb mode is excited in this spectral regime where the wavelength is much larger than the thickness of the plate, reducing greatly the complexity due to 
multi-mode conversion. Meanwhile, since we operate below the stop band, Bragg refraction is absent and relatively good transmission is achieved even when a double-step is added to the plate to better match isofrequency contours. Finally, this geometry opens the way to explore experimentally flat lens super-focusing by replacing the non-resonant holes by subwavelength resonant scatterers such as blind holes or pillars ${ }^{40}$.

\section{ACKNOWLEDGEMENTS}

M.D. acknowledges PhD funding from the Direction Générale de l'Armement (DGA).

S.G. is thankful for an ERC starting grant funding (ANAMORPHISM).

P. S. is thankful to the Agence Nationale de la Recherche support under grant ANR PLATON ${ }^{\circ}$ 12-BS09-003-01.

Supplementary Information accompanies this paper. 


\section{REFERENCES}

1 V.G. Veselago, Sov. Phys. Usp. 10 (4), 509-514 (1968)

2 J. B. Pendry, Phys. Rev. Lett. 85, 3966 (2000).

3 D. R. Smith, W. J. Padilla, V. C. Vier, S. C. Nemat-Nasser, and S. Schultz, Phys. Rev. Lett. 84, 4184 (2000).

4 S. A. Ramakrishna, Rep. Prog. Phys. 68, 449 (2005).

5 Z. Liu, X. Zhang, Y. Mao, Y. Y. Zhu, Z. Yang, C. T. Chan, P. Sheng, Science 289, 1734 (2000); P. Sheng, Physica B. 394, 256-261 (2007).

6 A. B. Movchan and S. Guenneau, Phys. Rev. B70,125116, (2004).

7 S. Guenneau, A.B. Movchan, S.A. Ramakrishna and G. Petursson, New Journal of Physics 9, 399 (2007).

8 J. Li and C.T. Chan, Phys. Rev. E 70, 055602 (2004).

9 N. Fang, D. Xi, J. Xu, M. Ambati, W. Srituravanich, C. Sun and X. Zhang, Nature Materials 5, 452 (2006); S. Zang, L. Yin, N. Fang, Phys. Rev. Lett. 102 (19): 194301 (2009).

10 M.S. Kushwaha, P. Halevi, L. Dobrzynski, and B. Djafari-Rouhani, Phys. Rev. Lett. 71, 2022-2025 (1993).

11 X. Hu, Y. Shen, X. Liu, R. Fu, and J. Zi, Phys. Rev. E 69, 030201R (2004).

12 M. Ke, Z. Liu, C. Qiu, W. Wang, and J. Shi, W. Wen and P. Sheng, Phys. Rev. B 72, 064306 (2005).

13 L. Feng, X.-P.Liu, M.-H.Lu, Y.-B.Chen, Y.-F.Chen, Y.-W.Mao, J. Zi, Y.-Y.Zhu, S.N.Zhu, and N.-B.Ming, Phys. Rev. Lett.96, 014301 (2006). 
14 M. Ke, Z. Li, Z. Cheng, J. Li, P. Peng, J. Shi, Solid State Communications 142, 177-180 (2007).

15 A. Sukhovich, L. Jing, and J. H. Page, Phys. Rev B 77, 014301 (2008).

16 A. Sukhovich, B. Merheb, K. Muralidharan, J. O. Vasseur, Y. Pennec, P. A. Deymier, and J. H. Page, Phys. Rev. Lett. 102, 154301 (2009).

17 J. Christensen, and F. J. García de Abajo, Phys. Rev. Lett. 108, 124301 (2012).

18 G. W. Milton, M. Briane, and J. R. Willis, New Journal of Physics 8, 248 (2006).

19 U. Leonhardt, T.G. Philbin, New J. Phys. 8, 247(2006).

20 M. Farhat, S. Guenneau, S. Enoch, A.B. Movchan, and G. Petursson, Appl. Phys. Lett. 96, 081909 (2010).

21 S. Timoshenko, Theory of Plates and Shells McGraw-Hill, New York, 1940.

22 K. F. Graff, Wave Motion in Elastic Solids Dover, New York, 1975.

23 C.H. Wang, L.R.F. Rose, in: D.O. Thompson, D.E. Chimenti (Eds.), Review of QNDE, vol. 22, AIP, New York, pp. 1615-1622 (2003).

24 P. Fromme, P. Wilcox, M.J.S. Lowe, P. Cawley, IEEE Trans. Ultra. Fer.Freq. Cont. 53, pp. 777-786 (2006).

25 A. B. Movchan, N. V. Movchan, and R. C. McPhedran, Proc. R. Soc. London, Ser. A 463, 2505 (2007).

26 N. Stenger, M. Wilhelm, and M. Wegener, Phys. Rev. Lett. 108, 014301 (2012) \& Viewpoint by R. McPhedran and A. Movchan in Physics 5, 2 (2012).

27 M. Brun, S. Guenneau and A.B. Movchan, Appl. Phys. Lett.94, 061903 (2009). 
28 M. Farhat, S. Guenneau, S. Enoch and A.B. Movchan, Physical Review B 79, 033102 (2009).

29 S. Bramhavar, C. Prada, A. A. Maznev, A. G. Every, T. B. Norris, and T. W. Murray, Phys. Rev. B 83, 014106 (2011).

30 J. Pierre, O. Boyko, L. Belliard, J. O. Vasseur, and B. Bonello, Appl. Phys. Lett. 97, 121919 (2010).

31 C. Luo, S. G. Johnson, J. D. Joannopoulos, and J. B. Pendry, Phys. Rev. B 65, 201104 (2002).

32 See supplementary material at [URL to be inserted by AIP] for a sketch of the anisotropic propagation in the platonic crystal responsible for the large angle negative refraction.

33 See supplementary material at [URL to be inserted by AIP] for more details on the analytic model used to derive Eq. 1.

34 The "free-space" bending-wave dispersion relation writes $p=c_{P} k^{2} h / \sqrt{12}$, where $p$ is the pulsation, $k$ the wavenumber of the flexural waves and $c_{p}=\left[\frac{E}{\rho\left(1-v^{2}\right)}\right]^{1 / 2}$ the plate-wave velocity, with $v$ the Poisson's ratio, $E$ the Young's modulus, $h$ the plate thickness and $\rho$ the density of the plate.

35 Elastic constants of Duraluminium $: E=74.9 \mathrm{GPa}, v=0.334, \rho=2790 \mathrm{~kg} / \mathrm{m} 3$.

36 See supplementary material at [URL to be inserted by AIP] for a movie of the measured time evolution of the pulse propagation and flat lens refocusing.

37 J. Virieux, Geophysics 51, 889-901 (1986). 
38 E. Bossy, M. Talmant, and P. Laugier, J Acoust. Soc. Am. 115, 2314-2324(2004). See also http://www.simsonic.fr/

39 R. W. Graves, Bulletin of the Seismological Society of America 86, pp. 1091 (1996).

40 M. A. Al-Lethawe, M. Addouche, A. Khelif and S. Guenneau, New J. Phys. 14, 123030 (2012).

41 R. Merlin, Appl. Phys. Lett. 84, 1290 (2004).

42 G. W.Milton, N. A. P. Nicorovici, R. C. McPhedran, \& V. A. Podolskiy, Proc. of the R. Soc. A 461, 3999 (2005). 


\section{FIGURES}
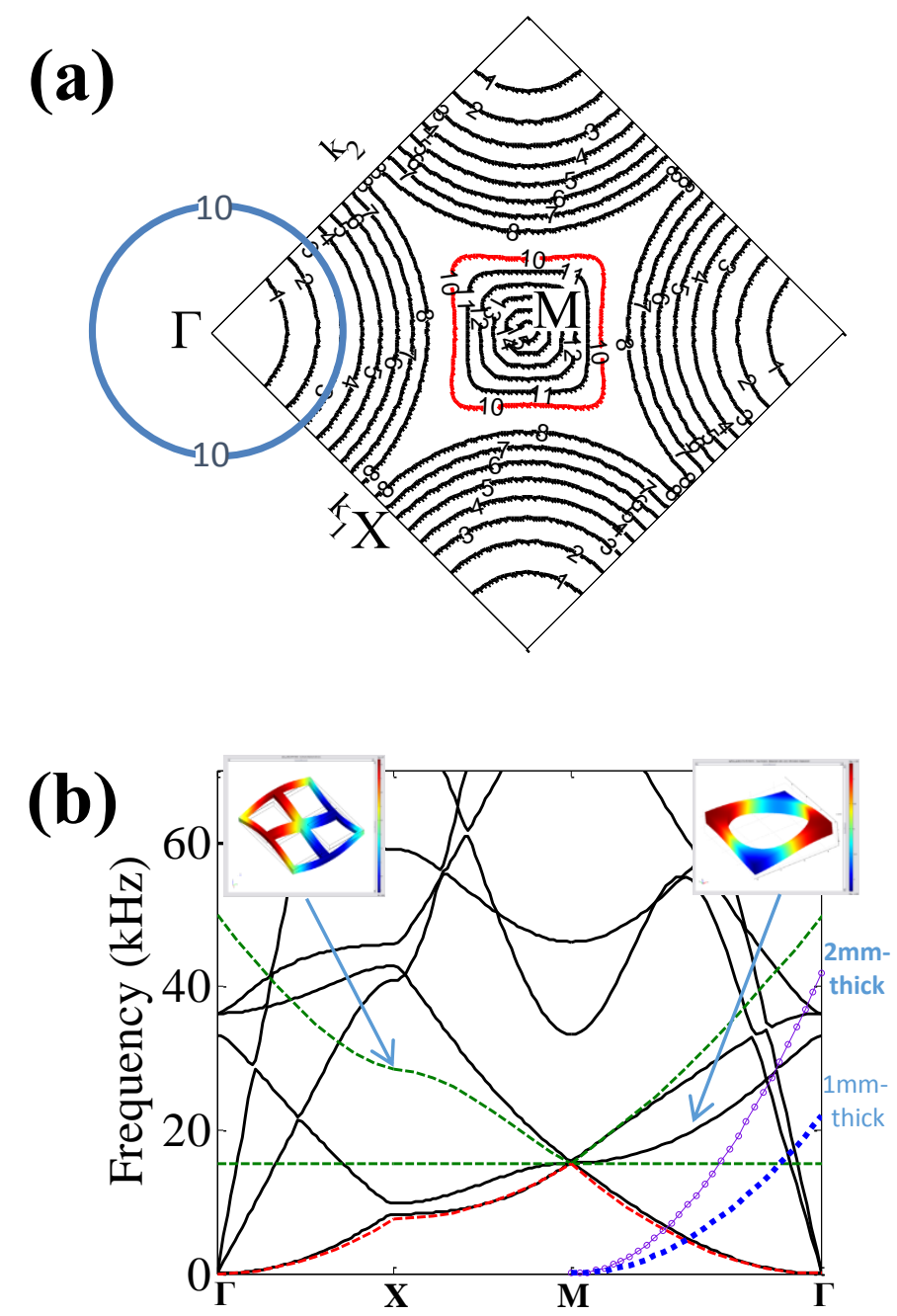

Figure 1: (a) Isofrequency contours. The $\left(\mathrm{k}_{1}, \mathrm{k}_{2}\right)$ plane has been rotated by $45^{\circ}$ to align the $\Gamma \mathrm{M}$-direction with the horizontal incident direction normal to the platonic lens. The crystal isofrequency at $10 \mathrm{kHz}$ circles the M-point (red circle) while the free-space isofrequency at $10 \mathrm{kHz}$ circles the Г-point (blue circle). (b) Band diagram. Horizontal axis: Projection of the Bloch vector on the boundary of the first irreducible Brillouin zone ГXM. Vertical axis: Bending wave frequency. Comparison of finite element computations (solid line) against analytical estimate given by Eq. 1 (dashed line). Excellent agreement is found in the acoustic band (dashed redline).The blue curves starting from the M-point correspond to "free space" bending waves propagating in $1 \mathrm{~mm}$-thick (full circles) and $2 \mathrm{~mm}$-thick (open circles) homogeneous plate along the ГM direction. Insets: unit cell of the actual platonic crystal (right) and for the analytical model (left). 


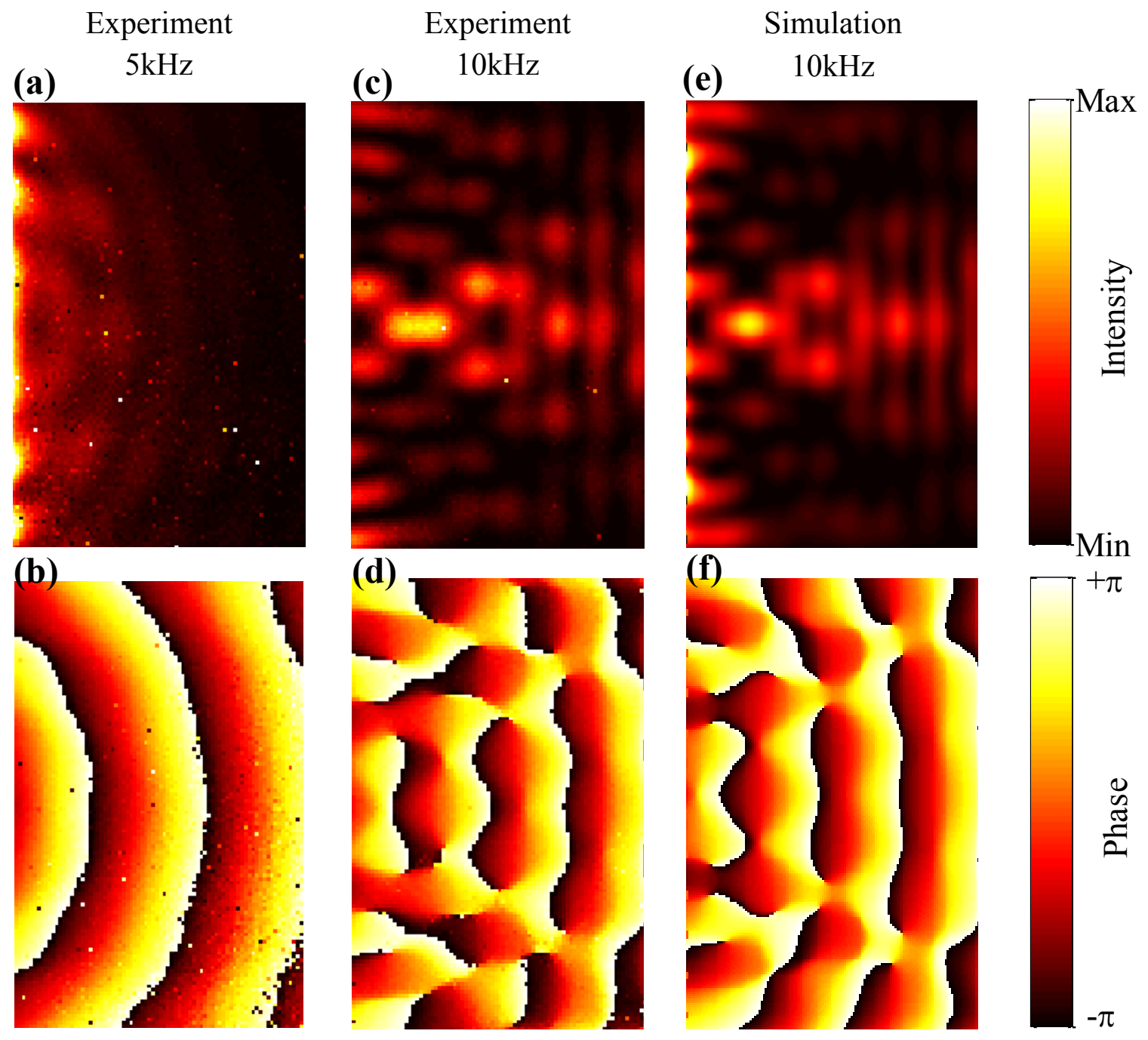

Figure 2 : Snapshot at time $t=833 \mu$ s of the amplitude squared (top) and phase (bottom) of the acoustic velocity field measured on the image side where focusing is expected (The full picture is available in the movie $\left.{ }^{36}\right)$. (a)\&(b): Experiment at $f_{0}=5 \mathrm{kHz}$ carrier frequency, which shows a divergent wavefront.(c)\&(d): Experiment at $f_{0}=10 \mathrm{kHz}$ carrier frequency, which shows refocusing. (f)\&(g): 3D-FDTD simulation also at $f_{0}=10 \mathrm{kHz}$. Note the velocity-field oscillations at the interface between the array of air holes and the homogeneous plate (left edge in (a),(c)\&(e)), which are one of the hallmarks of focusing through a negatively refracting lens $\mathrm{s}^{41,42}$. 


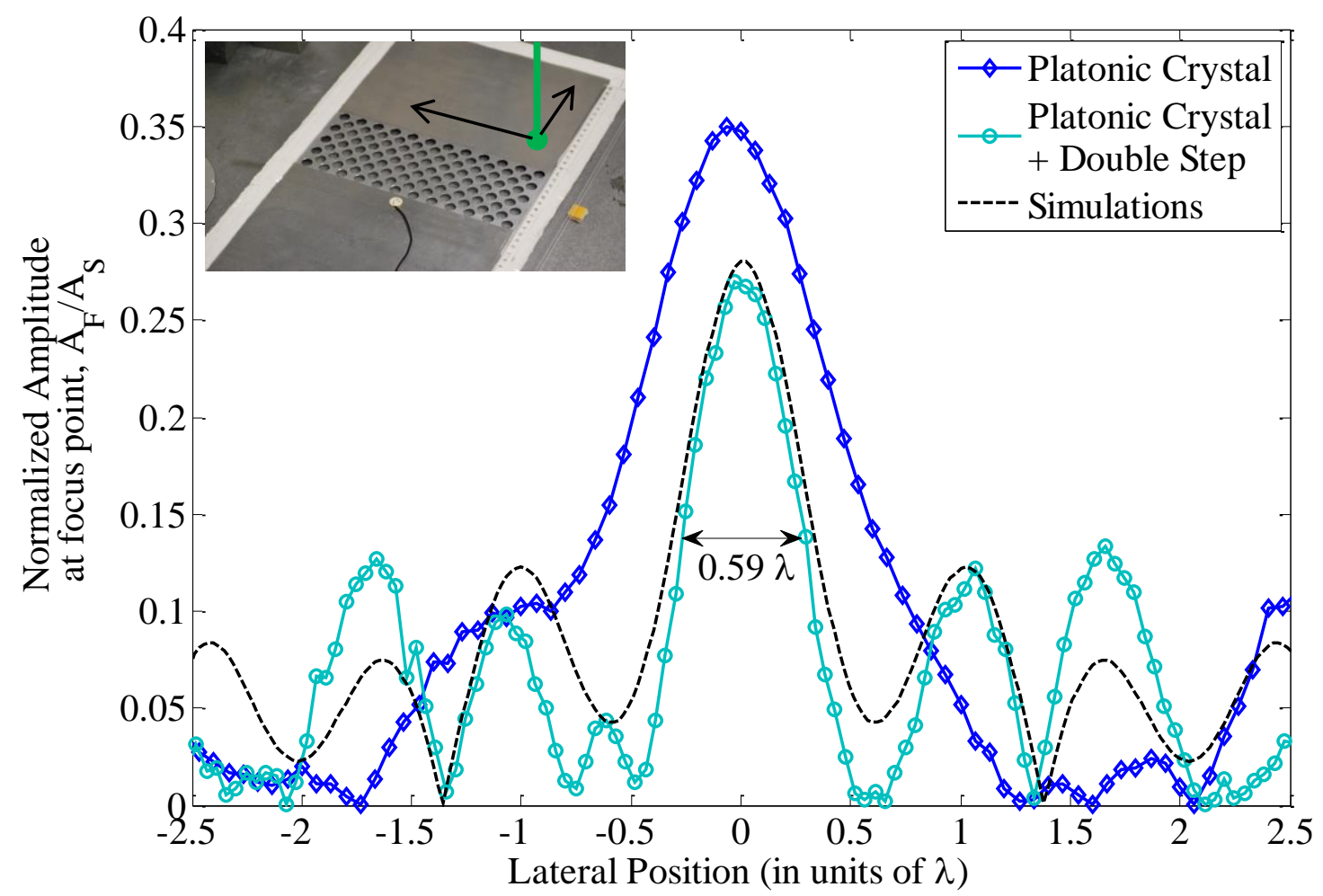

Figure 3: Normalized lateral magnitude of the acoustic velocity across the image point at $t=833 \mu$ s. Comparison between the lateral profile in the plate with constant thickness $1 \mathrm{~mm}$ (dark blue line with open diamonds) and in the plate with doublestep $(2 \mathrm{~mm}-1 \mathrm{~mm}-2 \mathrm{~mm})$ (cyan line with open circles). While the resolution given by the FWHM of the central peak is $1.0 \lambda$ in the uniform plate, it becomes $0.59 \lambda$ in the double-step structure, thus confirming the larger-angle negative-refraction achieved by matching free-space and platonic crystal dispersion curves. Similar resolution $(0.61 \lambda)$ is obtained through 3DFDTD simulations where absorbing layers similar to the experiment have been implemented (dashed black line). Amplitude has been normalized to the maximum of the input pulse. Position is in units of $\lambda$ measured in the plain plate. Inset: The double step ( $2 \mathrm{~mm}-1 \mathrm{~mm}-2 \mathrm{~mm}$ thick) rectangular plate with the square lattice of see-through circular $12 \mathrm{~mm}$-diam holes. The source, a piezoelectric pastille, is acoustically coupled to the metallic plate. Blue-tack is stuck on both sides and edges of the plate to damp the acoustic waves and reduce reflections. The green-light beam of the laser vibrometer in actuality scans the plate surface opposite the emitter. 